\title{
Stereoacuity and refractive, accommodative and vergence anomalies of South African school children, aged 13-18 years
}

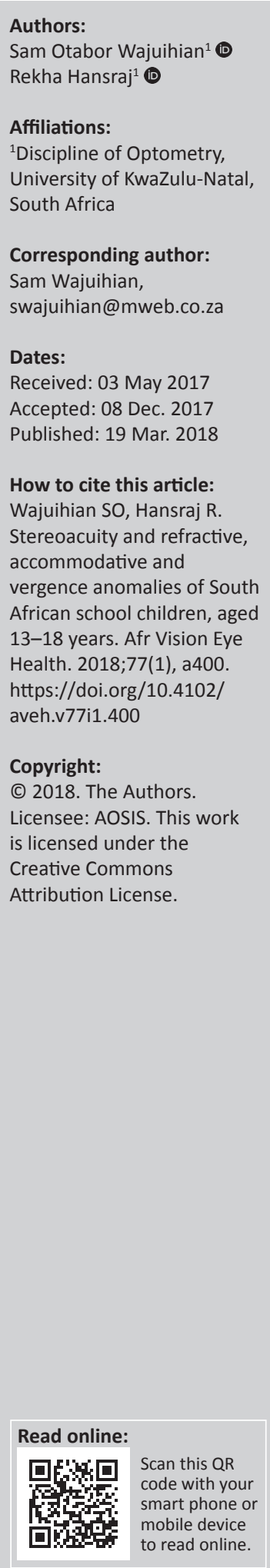

Aim: The aim of this study was to explore possible associations between stereoacuity and refractive, accommodative and vergence anomalies.

Methods: The study design was cross-sectional and comprised data from 1056 high school children aged between 13 and 18 years; mean age and standard deviation were $15.89 \pm 1.58$ years. Using a multi-stage random cluster sampling, participants were selected from 13 high schools out of a sample frame of 60 schools in the municipality concerned. In the final sample, $403(38 \%)$ were males and 653 (62\%) females. Refractive errors, heterophoria, near point of convergence, fusional vergences and accommodative functions (amplitude, facility, response and relative) were evaluated. Stereoacuity was evaluated using the Randot stereotest and recorded in seconds of arc where reduced stereoacuity was defined as worse than $40 \mathrm{~s}$ arc.

Results: Overall, the mean stereoacuities (in seconds of arc) of the children with anomalies were the following: those with refractive errors (52.6 \pm 36.9$)$, with accommodative anomalies $(53.1 \pm 34.1)$ and with vergence anomalies $(48.29 \pm 31.1)$. The mean stereoacuity of those with vergence anomalies was significantly better than that of those with either refractive errors or accommodative anomalies $(p=0.02)$. In the refractive error category, only anisometropia had significantly reduced mean stereoacuity compared to emmetropia (Mann-Whitney U: $p=$ 0.01). The mean stereoacuity of cases of accommodative anomalies was significantly reduced compared to those without such anomalies (Mann-Whitney $\mathrm{U}: p=0.01$ ). Similarly, the mean stereoacuity of cases with vergence anomalies was significantly reduced compared to those without vergence anomalies $(p=0.02)$.

Conclusion: Refractive errors, accommodative or vergence anomalies are more likely to have reduced mean stereoacuity than cases without such anomalies. Refractive errors or accommodative anomalies had significantly more reduced stereoacuity than vergence anomalies. These findings suggest that the Randot stereotest could be used to identify those with such anomalies, and this study extends knowledge regarding the possible use of stereoacuity as a useful tool to screen for binocular anomalies.

\section{Introduction}

Stereopsis, described as binocular visual depth perception based on retinal rivalry, is one of the parameters used to evaluate the interactions of the accommodative and vergence systems. ${ }^{1,2}$ Stereoacuity is the clinical measure of stereoscopic threshold derived from the minimum disparity that results in the appreciation of depth and it is an indicator of binocularity. ${ }^{1,2}$ Accommodative anomalies are disorders of the eye's focussing mechanism that result in an inappropriate response to a particular visual demand and are classified into accommodative insufficiency (AI), accommodative excess (AE) and accommodative infacility (AIF) ${ }^{3,4}$ Near point vergence anomalies are disorders of binocular vision that cause an inability to sustain comfortable bifoveal fixation ${ }^{5}$ and include convergence insufficiency (CI), convergence excess (CE) and fusional vergence dysfunction (FVD). ${ }^{5}$

The refractive error, accommodative and vergence mechanisms are all part of the visual efficiency system. ${ }^{3}$ During fusion, decreased visual acuity (VA), uncorrected refractive error as well as sensory and motor fusion anomalies are thought to influence stereoscopic ability. ${ }^{2,6,7}$ Depending on the disparity of retinal images, the refractive error and accommodative system maintain focus, while the vergence system fuses the images formed about the foveae of the two eyes to further ensure single vision. ${ }^{8}$ Viewing a stimulus at near requires adequate interaction of the visual efficiency mechanisms that minimise the blur and disparity of retinal images. ${ }^{8}$ The constant 
interactions between these near task mechanisms increase the difficulty in maintaining accommodative-vergence balance. Consequently, a breakdown of the system could result in manifestation of symptoms of asthenopia that may affect visual efficiency and academic performance in school children. ${ }^{3,5,8}$

Poor stereoacuity is associated with vision anomalies as well as with reading difficulties in kindergarten and firstgrade school children. ${ }^{6}$ Besides its use as a clinical tool for detecting anomalies in the visual system, good stereoacuity is associated with better long-term stability of alignment and reduced risk for, and lesser severity of, amblyopia, improved achievement of sensorimotor developmental milestones, efficient reading ability and improved quality of life. ${ }^{9}$ Given the clinical importance of the near point mechanism, various studies $6,7,8,9,10,11,12,13,14,15,16,17,18,19,20,21,22,23,24,25$ have investigated associations of stereoacuity with various anomalies although most of these studies $7,10,11,12,13,14,15,16,17,18,19,20,21,22,23,24,25$ focussed mainly on the association between poor stereoacuity and refractive errors, while empirical studies on the distribution of reduced stereoacuity and possible association between stereoacuity and accommodative-vergence anomalies could not be found.

Investigating the association of reduced stereoacuity with refractive, accommodative and vergence anomalies would provide information on the possible use of stereotests to screen for binocular anomalies. Therefore, the aim of the present study was to explore the association of these anomalies with stereoacuity. In comparison with previous studies, a unique aspect of our study is that we investigated correlations of stereoacuity with clinical measures of refractive, accommodative and vergence functions, which were all identified using standardised classification. In addition, the means and percentages of stereoacuity were compared between cases with refractive, accommodative or vergence anomalies and those without such anomalies. The hypothesis tested was that reduced stereoacuity among cases with anomalies compared to those without such anomalies would suggest that the Randot stereoacuity test could be useful to screen for and identify these anomalies.

\section{Methods \\ Study design}

This report is part of a larger cross-sectional study designed to quantify near vision anomalies (refractive and accommodativevergence) and its association with symptoms in order to develop strategies on how to identify and treat them.

\section{Participants and study setting}

The target population was black high school children in the uMhlathuze Municipality of northern Kwazulu-Natal Province, South Africa. The participants for the study comprised 1211 children (481 males and 730 females) with age ranges of between 13 and 18 years who were selected from 13 out of a sample frame of 60 high schools in the municipality. The sample size was derived from the original study - part of which has been published. ${ }^{5,26,27}$ Information sheets and consent forms were written in both English and the children's indigenous language (isiZulu). Only black South African children of either gender were eligible to participate in the study. In the identified schools, assent forms were distributed to selected students and consent forms for parents were sent to them via their children. The leaflets contained information explaining the purpose of the study. Students who returned their approved parental consent and assent forms were enrolled for the study which also complied with the Declaration of Helsinki regarding research on human subjects. ${ }^{5}$ For the entire study, students were excluded if they had suppression on the Worth-4-dot, strabismus, ocular diseases, nystagmus, motility problems, any systemic conditions or were on any systemic medication that might affect near vision function.

\section{Materials and procedure}

The school principals provided rooms at the school venues where the visual examinations were conducted. The purpose and procedure for every technique were carefully explained to each participant before starting the eye examination. Validated optometric instruments were used and techniques were applied as described in our previous publications. ${ }^{5,26,27}$ The eye examination techniques comprised two main stations. All vision testing was performed between 08:30 and 13:30. As much as possible, the same conditions including test distances and standard room illumination were applied for all sessions and at all testing sites.

\section{Eye examination procedures}

The testing sequence was arranged in two stations. The techniques performed in the first station by a trained assistant included case history comprising recording of each participant's demographic details, VA measurement and history of ocular and systemic conditions. To minimise bias, the research assistant in station 1 worked independently of the optometrist who collected data in station 2 .

The tests in the second station were performed by one optometrist only who was experienced in performing the techniques, therefore eliminating any concerns of interexaminer variability. The techniques performed in the second station are as described in our previous publications. ${ }^{5,26,27}$ The preliminary tests were performed with participants wearing no refractive correction and included ocular health status evaluation using the direct ophthalmoscope, and suppression was evaluated at near using the Worth-4-dot test (Bernell Corporation, Mishawaka Inc, IN, USA)., ${ }^{4,26,27}$ Other clinical assessments included ocular motility using the Broad-H-test and stereoacuity, which was assessed using the Randot stereotest (Vision Assessment Corporation, IL, USA) without refractive correction, ${ }^{18}$ as an objective of the study was to compare stereoacuity of those with refractive errors and those with emmetropia. The Randot stereotest was 
performed at $40 \mathrm{~cm}$ in normal room illumination with each participant seated and wearing vectographic spectacles. Failure or limit of disparity was based on the first incorrect response on the circles except when the patient identified two consecutive finer stimuli correctly. ${ }^{4}$

Refractive error was determined objectively (without cycloplegia) with an autorefractor (MRK/3100; Huvitz) and refined subjectively using the phoropter. ${ }^{4,26,27}$ Cycloplegia could not be applied as the entire study was on investigating near vision anomalies and cycloplegia could affect such near vision functions.

Accommodative and vergence tests were performed at $40 \mathrm{~cm}$ with the best refractive compensation in place. The tests were performed three times and an average reading taken for analysis. For vergence parameters, the near point of convergence (NPC) was measured using the Royal Air Force (RAF) rule with a vertical line target. The unilateral cover test was first performed to rule out strabismus, while distance and near heterophoria were evaluated using the von Graefe technique in a specially designed phoropter tripod (Figure 1) and fusional vergences (FVs) were measured using horizontal prism bars.

For accommodative measures, the amplitude of accommodation (AA) was assessed using the Donder's push-up-to-blur method with the RAF near point rule. ${ }^{5,26,27}$ The accommodation response (AR) was measured using the monocular estimated method (MEM) dynamic retinoscopy technique. Accommodative facility (AF) was assessed using \pm 2 D flipper lens or lenses. Relative accommodation was measured using the phoropter and negative relative accommodation (NRA) was measured first, followed by positive relative accommodation (PRA). Positive or negative lenses were added for NRA or PRA, respectively.5,26,27 Children identified with anomalies were referred for further assessments.

\section{Data analysis}

The Statistical Package for Social Sciences (SPSS) version 21 was used for analysis. Descriptive statistics included means, standard deviations and medians. Kolmogorov-Smirnov (K-S) tests were used to test for normality of data. The chisquared and logistic regressions tests were performed to test for differences in proportions for categorical variables among groups. For means, the Mann-Whitney (MW) and the Kruskal-Wallis (KW) equality-of-population rank nonparametric tests were used to compare differences in means between and among groups. Distributions of variables were presented using tables and proportions and corresponding 95\% confidence intervals were presented with prevalence. A significance level of less than 0.05 was considered significant.

All outcome variables were classified based on previous reports and refractive errors, ${ }^{27}$ accommodative, ${ }^{26,28}$ vergence anomalies $^{5}$ and heterophoria ${ }^{29}$ were classified based on criteria used in other studies (Table 1). Normal stereoacuity was defined as stereoacuity better than $40 \mathrm{~s}$ arc, while reduced stereoacuity was defined as stereoacuity worse than $40 \mathrm{~s}$ arc. ${ }^{3}$ In stereoacuity interpretation, higher stereoacuity numbers mean worse or reduced thresholds, while lower numbers imply better thresholds. ${ }^{3}$

\section{Ethical considerations}

The study protocol was approved by the Biomedical Research Ethics Committee (ethical clearance reference number BE $177 / 12$ ) of the University of KwaZulu-Natal, South Africa. Written informed consents for access to the schools were obtained from the Department of Education and the concerned school principals.

\section{Results \\ Sample characteristics}

A total of 1211 children who returned their consent and assent forms were included in the study, but 10 were excluded (seven had amblyopia, one had diabetes, another had glaucoma and the other had corneal scars because of trauma), leaving a sample of 1201 . Thereafter, a further 145 children aged 19 years and above were excluded from this report as we intended to report only on participants of official high school age range of 13-18 years. Thus, data were analysed for a total sample of 1056 participants (a response rate of $87 \%$ ), with mean age $15.89 \pm 1.58$ years and median age 16 years; $403(38 \%)$ were males and $653(62 \%)$ were females. The sample comprised $781(73.96 \%)$ students from grades 8-10 (lower grade level) and 275 (26.07\%) from grades 11 and 12 (higher grade level).

\section{Descriptive statistics for all variables}

Only data for near distance results are reported and data for most variables were not normally distributed and participants analysed had normal near VA. Table 2 shows the descriptive statistics for the right eye (RE) visual acuities and refractive error (RE), NPC, heterophoria (phoria) and FVs. Given the moderate positive correlation between the distance left and

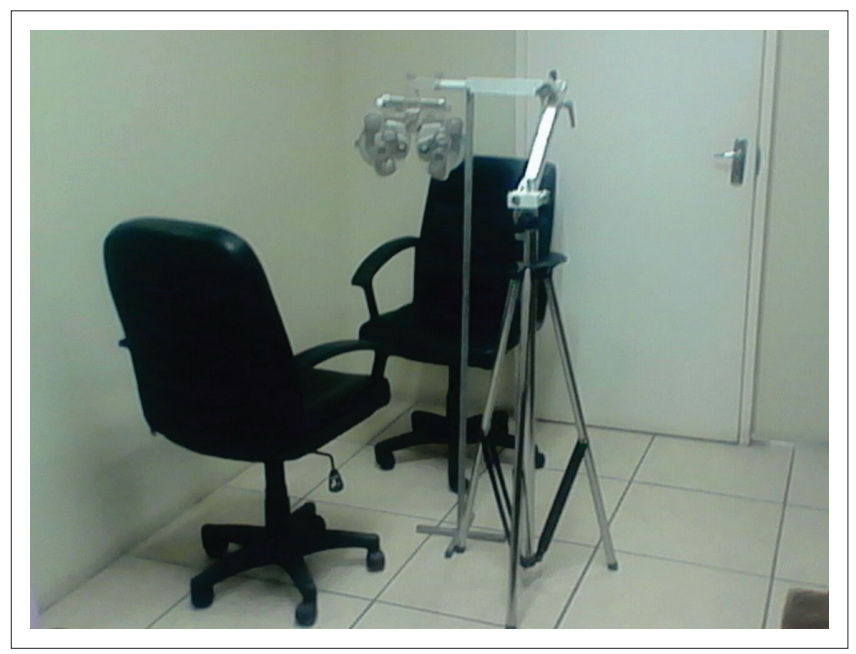

Source: Photo taken by Samuel O. Wajuihian

FIGURE 1: A phoropter head attached to a specially designed tripod stand. The setup is ideal for vision screening outreaches. 
TABLE 1: Classification criteria for anomalies in this study.

\begin{tabular}{|c|c|c|}
\hline Variables & Anomalies & Criteria \\
\hline \multirow[t]{4}{*}{ Refractive error } & Myopia & $\leq-0.50 \mathrm{D}$ \\
\hline & Hyperopia & $\geq 0.50 \mathrm{D}$ \\
\hline & Astigmatism & $\leq-0.75 \mathrm{D}$ \\
\hline & Anisometropia & At least $-0.75 \mathrm{D}$ difference between both eyes spherical equivalent (sphere $+1 / 2$ cylinder) \\
\hline \multirow[t]{11}{*}{ Accommodative anomalies } & Accommodative insufficiency & Minimum of clinical signs (1) and one other or all clinical signs: \\
\hline & & $\begin{array}{l}\text { (1) Reduced AA. Push-up monocular AA at least } 2 \mathrm{D} \text { below Hofstetter's calculation for minimum amplitude: } \\
15-0.25 \text { (age in years). }\end{array}$ \\
\hline & & (2) High values on MEM retinoscopy: > $0.75 \mathrm{D}$. \\
\hline & & (3) Fails monocular AF testing ( $<6$ cycles per minute) with $\pm 2 \mathrm{D}$. \\
\hline & Accommodative excess & Clinical signs (1) and (2) or (1) and (3): \\
\hline & & (1) Low MEM $<0.25$ \\
\hline & & (2) Difficulty clearing $2 \mathrm{D}(<6$ cycles per minute) with MAF. \\
\hline & & (3) Fails binocular accommodative facility (BAF) test (<3 cycles per minute) with $2 \mathrm{D}$. \\
\hline & Accommodative infacility & Clinical signs (1) and (2) or (1) and (3): \\
\hline & & (2) Positive relative accommodation (PRA) $\leq|-2 \mathrm{D}|$. \\
\hline & & (3) Negative relative accommodation (NRA) <+2.50 D. \\
\hline \multirow[t]{12}{*}{ Vergence anomalies } & Convergence insufficiency & (1) Exophoria at near. \\
\hline & & (2) Exophoria at near $\geq 4$ prism dioptre $(\mathrm{pd})$ and greater than the distance phoria. \\
\hline & & $\begin{array}{l}\text { (3) Insufficient fusional vergence: (a) fails Sheard's criteria or (b) poor PFV at near } \leq 12 \mathrm{pd} \text {. Base out (BO) to } \\
\text { blur or } \leq 15 \mathrm{pd} \text { BO break. Poor BO break was used for PFV criteria. }\end{array}$ \\
\hline & & (4) Receded NPC $\geq 7.5 \mathrm{~cm}$ break or $\geq 10.5 \mathrm{~cm}$ recovery. \\
\hline & Convergence excess & Minimum of two clinical signs below: \\
\hline & & (1) Significant esophoria at near $\geq 2 \mathrm{pd}$. \\
\hline & & (2) Reduced NFV at near $<8 / 16 / 7$ for blur/break/recovery ( 1 of 3 ). \\
\hline & & (3) High MEM ( $\geq+0.75)$ (may show high lag). \\
\hline & Fusional vergence dysfunction (FVD) & (1) Reduced fusional vergences, (2) Normal phoria, (3) minimal refractive error \\
\hline & Heterophoria (prism dioptres $(\mathrm{pd})$ & Orthophoria: no deviation \\
\hline & & Exophoria: any magnitude of exo-deviation \\
\hline & & Esophoria: any magnitude of eso-deviation \\
\hline
\end{tabular}

Source: Wajuihian SO, Hansraj R. Association of symptoms with refractive, accommodative and vergence anomalies in high school children in South Africa. Optom Vis Perf. 2017;5(1):27-36 $\mathrm{AF}$, Accommodative facility; MAF, monocular accommodative facility; BAF, binocular accommodative facility; NPC, near point of convergence; NFV, negative fusional vergence; PFV, positive fusional vergence; $\mathrm{BO}$, base out; $\mathrm{BI}$, base in; MEM, monocular estimation technique.

right eyes (spherical equivalents) $(r=0.64, p=0.01)$, only the results for the right eyes are included here although anisometropia was diagnosed as differences between both eyes in terms of their spherical refractions.

\section{Associations of stereoacuity with refractive, accommodative and vergence anomalies}

For all anomaly categories, the means and percentages (\%) of stereoacuity were compared for cases with anomalies to those without anomalies, that is, cases of refractive errors versus emmetropia; vergence anomalies versus no vergence anomalies and accommodative anomalies versus no accommodative anomalies (Table 3). The comparisons were made for both the entity and individually, for example, total refractive errors with emmetropia, as well as emmetropia versus the respective refractive error (myopia, astigmatism, hyperopia or anisometropia). Only the data and inferences on mean stereoacuity are shown in the table - the percentages are not shown.

For this report, only the stereoacuity (in seconds of arc) measured without refractive correction was presented. For the total sample, $496(41.30 \%)$ of participants had reduced (> 40 s arc), while 705 (58.7\%) had normal stereoacuity. Overall, the mean stereoacuity of those with either refractive errors or those with accommodative anomalies were significantly more reduced than for those with vergence anomalies $(p=0.02)$.

\section{Refractive error}

Comparing the mean stereoacuity of those with refractive errors (52.6 $\pm 36.9 \mathrm{~s}$ arc) with that for those with emmetropia $(42.7 \pm 0.49 \mathrm{~s} \mathrm{arc})$, the KW test revealed that those with refractive error had significantly reduced stereoacuity compared to those with emmetropia ( $p=0.01$, Table 3 ).

Comparing the mean stereoacuity of those with refractive errors with that of those with emmetropia, only anisometropia was significantly associated with reduced stereoacuity.

Using percentages, those with anisometropia (68\%) or myopia (50\%) had the highest percentages of reduced stereoacuity, while those with hyperopia $(45 \%)$ or emmetropia $(39 \%)$ or astigmatism had better stereoacuity (32\%). But myopia $(p=0.17)$, hyperopia $(p=0.48)$ and astigmatism $(p=0.31)$ were not significantly associated with reduced stereoacuity, whereas those with anisometropia were significantly more likely to present with reduced stereoacuity than emmetropia (OR 0.31, $p=0.01$, logistic regression). However, those with emmetropia were significantly more likely to present with normal stereoacuity than for those with myopia $(\mathrm{OR}=1.720, p=0.02$, logistic regression). 
TABLE 2: Descriptive statistics for stereoacuity and refractive errors, accommodative and vergence measures in school-aged children ( $N=1056)$, aged $13-18$ years.

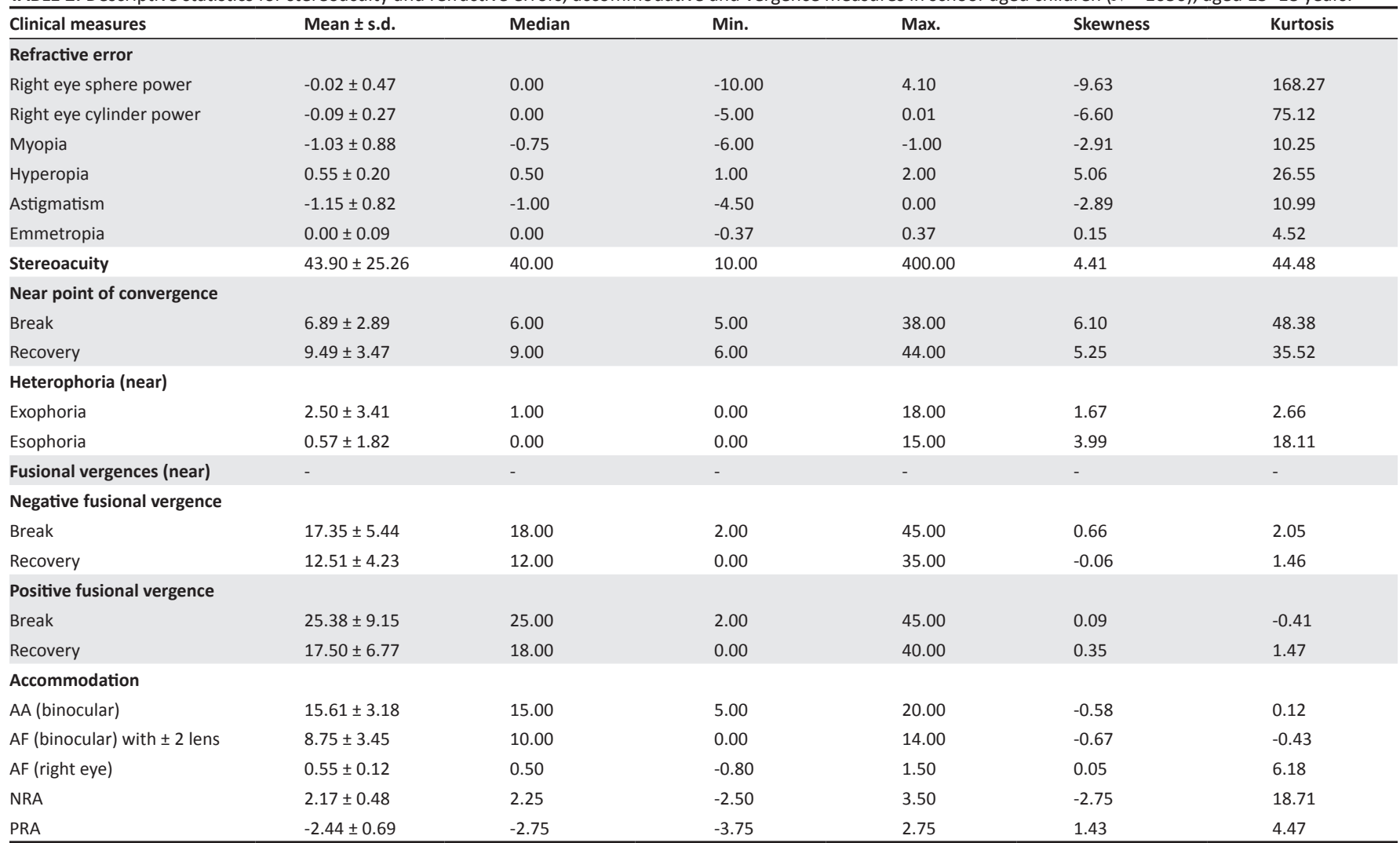

AA, amplitude of accommodation; AF, accommodative facility; NRA, negative relative accommodation; PRA, positive relative accommodation.

TABLE 3: Mean, standard deviation and median stereoacuities for those with refractive errors, or accommodative, or vergence anomalies or no anomalies (emmetropia or no vergence anomalies or no accommodative anomalies).

\begin{tabular}{|c|c|c|c|c|c|c|}
\hline \multirow[t]{2}{*}{ Clinical measures } & \multirow[t]{2}{*}{$n$} & \multirow[t]{2}{*}{ Prevalence $(\%)$} & \multicolumn{2}{|c|}{ Stereoacuity (s arc) } & \multirow[t]{2}{*}{ MWU ( $p=)$} & \multirow[t]{2}{*}{ Median } \\
\hline & & & Mean & s.d. & & \\
\hline Refractive errors & - & - & 52.64 & 36.90 & - & - \\
\hline Astigmatism & 25 & 2.3 & 42.76 & 30.49 & 0.12 & 32 \\
\hline Anisometropia & 16 & 1.3 & 75.04 & 86.93 & 0.01 & 56 \\
\hline Hyperopia & 72 & 6.8 & 47.91 & 28.09 & 0.10 & 40 \\
\hline Myopia & 63 & 6.0 & 45.28 & 16.52 & 0.07 & 50 \\
\hline Emmetropia $\dagger$ & 896 & 84.9 & 42.77 & 22.49 & - & 40 \\
\hline Totals & 1056 & 100.0 & - & - & - & - \\
\hline Vergence anomalies & - & - & 48.29 & 31.16 & - & - \\
\hline Convergence insufficiency & 233 & 22.0 & 45.85 & 36.05 & 0.02 & 40 \\
\hline Convergence excess & 60 & 5.6 & 56.76 & 30.73 & 0.02 & 50 \\
\hline Fusional vergence dysfunction & 33 & 3.1 & 50.23 & 26.71 & 0.02 & 50 \\
\hline No vergence anomalies & 730 & 69.1 & 42.03 & 23.13 & 0.02 & 40 \\
\hline Totals & 1056 & 100.0 & - & - & - & - \\
\hline Heterophoria (near) & - & - & 47.24 & 32.40 & - & - \\
\hline Orthophoria & 387 & 36.6 & 45.32 & 25.27 & - & 40 \\
\hline Exophoria & 546 & 51.7 & 43.88 & 24.35 & 0.76 & 40 \\
\hline Esophoria & 123 & 11.6 & 52.54 & 47.60 & 0.01 & 40 \\
\hline Totals & 1056 & 100.0 & - & - & - & - \\
\hline Accommodative anomalies & - & - & 53.10 & 34.14 & - & - \\
\hline Accommodative insufficiency & 51 & 4.8 & 59.12 & 34.82 & 0.01 & 50 \\
\hline Accommodative excess & 32 & 3.0 & 50.16 & 31.14 & 0.11 & 50 \\
\hline Accommodative infacility & 137 & 12.9 & 51.81 & 36.46 & 0.01 & 50 \\
\hline No accommodative anomalies & 836 & 79.1 & 42.56 & 23.81 & 0.01 & 40 \\
\hline Totals & 1056 & 100.0 & - & - & - & - \\
\hline
\end{tabular}

MWU, Mann-Whitney test or statistic.

$\dagger$, The mean for emmetropia was compared to that of the respective refractive error: myopia, hyperopia, astigmatism and anisometropia.

$p$-values with significant differences are highlighted in bold.

Bold numbers (other than $p$-values) $=$ main reference values. 


\section{Vergence anomalies}

Participants with vergence anomalies (CI, CE or FVD) had significantly reduced mean stereoacuity compared to cases without vergence anomalies (MW, $p=0.02$, Table 3).

Using percentages, there was no significant association between vergence anomalies and reduced stereoacuity (CI: $p=0.72$; CE: $p=0.14$ and FVD: $p=0.33$ ), but this is not shown in the table.

The mean stereoacuity of esophoria was significantly reduced compared to cases with orthophoria $(p=0.01)$, and there were significant correlations between reduced stereoacuity and near exophoria $(r=0.130, p=0.01)$ and near esophoria $(r=0.185, p=0.01)$, but this is not shown in the table.

\section{Accommodative anomalies}

The mean stereoacuity of those with accommodative anomalies was significantly reduced compared to cases without accommodative anomalies (MW, $p=0.01$, Table 3). Accommodative insufficiency and AIF had significantly reduced mean stereoacuity ( $p=0.01$ for either), while AE was not significantly associated with reduced stereoacuity ( $p=0.11$, Table 3$)$. For percentages, AI, AIF and AE were significantly associated with reduced stereoacuity (AI: $\chi^{2}=$ 20.31, $p=0.01$; AIF: $\chi^{2}=8.57, p=0.03$ and AE: $\chi^{2}=4.94$, $p=0.02)$.

\section{Discussion}

We explored possible associations of refractive error or accommodative or vergence anomalies with stereoacuity in a sample of 1056 school children. The major finding of this study is that those with anisometropia, AI, AIF or vergence anomalies had significantly reduced stereoacuity compared to cases without such anomalies. This suggests that the Randot stereotest could be useful to identify those with uncompensated refractive error, or accommodative or vergence anomalies.

In a study on pre-schoolers, Ciner et al. ${ }^{30}$ found that vision disorders, including refractive errors, were associated with reduced stereoacuity and severe vision disorders were more likely associated with poorer stereopsis than milder or no vision disorders. Trachimowicz et al. ${ }^{12}$ found high levels of uncorrected myopia (approximately -5 D or more) significantly impacted performance on stereotests in a sample of university students. Similarly, we found an increased likelihood of myopia having reduced stereoacuity than emmetropia although all myopia in our study was low (<-3 D). In contrast, Gawecki and Adamski ${ }^{19}$ found that hyperopes had the poorest stereoacuity and Ips et al. ${ }^{13}$ and Kulp et al. ${ }^{14}$ found moderate-to-severe hyperopia to be significantly associated with reduced stereoacuity. They emphasised that mainly greater magnitudes of hyperopia are associated with reduced stereoacuity even in children with non-strabismic disorders. ${ }^{14}$ In the present study, all cases of hyperopia in the participants were relatively mild (0.50-2 D) and not significantly associated with reduced stereoacuity in contrast to the findings by Kulp et al. ${ }^{14}$ Similar to our findings on anisometropia, Yang et al. ${ }^{15}$ and Robaei et al. ${ }^{18}$ found only anisometropia to be significantly associated with reduced stereoacuity. It has been suggested that anisometropia is more likely to affect stereoacuity because of more retinal disparities, associated aniseikonia and foveal suppression in the defocused eye.,22 Blur and reduced monocular or binocular VA from uncompensated refractive errors affects stereoacuity, with even small amounts of blurring affecting stereoscopic thresholds greatly., 2,15,21 The blur caused by refractive anomalies reduces the precision of stereoacuity by decreasing the disparity detectors' ability to detect and process horizontal disparities. ${ }^{2,31}$ With normal ocular pupils, errors of $1 \mathrm{D}$ or $2 \mathrm{D}$ could cause nearly 2- and 10-fold reductions in stereoacuity, respectively. ${ }^{31}$

We found a significant association between accommodative anomalies and reduced stereoacuity. Approximately $8.6 \%$ of participants with AIF had reduced stereoacuity, whereas Garzia and Nicholson ${ }^{23}$ found that $6 \%$ of those with poor AF failed their stereoacuity tests and Daum ${ }^{32}$ also found reduced stereoacuity in AI. The stimuli for accommodation include blur, retinal disparity and awareness of proximity. ${ }^{33}$ The blur caused by an accommodative error reduces the precision of stereopsis. ${ }^{31}$

The literature is scanty on the relationship of vergence anomalies with stereoacuity and the findings on their possible association are inconclusive. We also found no association between those with vergence anomalies and reduced stereoacuity on percentages even though the means of the vergence anomalies (CI, CE or FVD, Table 3) had significantly reduced stereoacuity compared with cases without such vergence anomalies. Such difference in significance levels may be related to criteria applied to define such anomalies and warrant further investigation. Patients with CI could have normal ( $40 \mathrm{~s}$ of arc or better) ${ }^{34}$ or reduced stereoacuity ${ }^{35}$ and, for example, Ponsonby et $a .^{6}$ found that children with reduced stereoacuity had a higher frequency of symptoms reported on the convergence insufficiency symptom survey.

Vergence eye movements align the eye and facilitate sensory fusion $^{36}$ and retinal disparity is the stimulus to both FV and stereopsis. ${ }^{36}$ In stereopsis, however, fusion of retinal disparities occurs between corresponding retinal elements within Panum's fusional area, while fusion of retinal disparity for vergence eye movement occurs outside the Panums fusion area. ${ }^{36}$ The complex interactions between sensory and the oculomotor processes that act to reduce excessive retinal disparity suggest a strong link between depth perception and motor fusion. ${ }^{37}$ Although stereoacuity does not depend directly on eye movements, given the strong link between them, it may be logical to expect that if the mechanisms that control vergence are impaired, stereoacuity may also be impaired..$^{38,39}$ The presence of vergence anomaly has been suggested to be associated with the occurrence of stereoanomaly. ${ }^{38,39,40}$ Therefore, it may be argued that vergence anomalies do seem to affect stereoacuity, at least up to a critical level ${ }^{38,39}$ or it may be that such effects may be restricted 
to coarse stereopsis (depth information derived from larger disparities that produce diplopia). ${ }^{40,41}$ The possible mechanism for reduced stereoacuity under vergence stress includes temporary anomalous retinal correspondence, which are small shifts in retinal correspondence that can occur without disrupting Panum's fusional process. ${ }^{42}$

A significant correlation between near lateral phoria and stereoacuity corroborates findings of other studies that found that even small amounts of esophoria negatively impact stereoacuity more than exophoria. ${ }^{2,24}$ However, Castren et al. ${ }^{11}$ found no correlation between heterophoria and stereoacuity although they excluded cases of high esophoria in their study, which may have influenced their findings. A possible mechanism for the associations between stereopsis and horizontal phoria could be that differences in stereo functions between the esophores and exophores may be related to relative strengths of the separate slow vergence adaptation mechanisms and the relation between fixation disparity and stereopsis. ${ }^{1,2}$ Exophores tend to have much smaller fixation disparities and could tolerate much larger amounts of heterophoria without developing symptoms than could esophores. ${ }^{1,2}$

In general, although stereoacuity is considered a benchmark and relatively simple procedure to measure clinical performance of binocular vision,,$^{1,2}$ the relationship between the vergence and stereoacuity functions is not always straightforward. ${ }^{1,2}$ Heterophoria requires FV efforts to maintain single binocular vision and involves the accommodative-convergence mechanism when viewing near targets. ${ }^{36,43}$ Fusional convergence may not be achieved if the eyes deviate excessively, even if the necessary retinal disparity control mechanisms exist in the brain. ${ }^{44,45}$ Similarly, stereopsis and binocular fusion cannot normally be expressed if the ocular deviation is excessive or if convergence does not allow bifixation at a near. ${ }^{44,45}$ Consequently, adequate refractive status, accommodation and optimal ocular alignment are required for good stereoacuity. Absence of adequate binocularity could result in poor stereoacuity and failure on the stereotest. Stereoacuity testing is also an important vision screening tool and children with normal stereoacuity are less likely to have marked difficulty with refractive or binocular vision anomalies. ${ }^{246}$ However, it does not necessarily imply that those with abnormal stereoacuity results will always have refractive and binocular vision anomalies but suggests the need for further evaluation. ${ }^{2}$

\section{Study limitations, strengths, implication and application}

Although applied in some studies (cited in our earlier articles, e.g., see Ref. 5), using the standard von Graefe technique to assess heterophoria and FV would have allowed for consistency in approach. However, we used von Graefe and prism bars (as in our pilot study, ${ }^{47}$ we confirmed that the children would have difficulties with rotary prisms to measure FVs in a school setting). However, the possible influences of this change in measurement methodology are unknown.
But the prism bar is a preferred tool in a screening setting as it is quicker and allows for easy viewing of eye movements and the modified method is easier for school-aged children to perform and understand in terms of their instructions. ${ }^{3}$

The present study has implications and applications in research and clinical practice towards identifying binocular anomalies. Our findings mean that the variables with significant associations with stereoacuity anomalies including anisometropia, AI, AIF as well as vergence anomalies could be identified using Randot stereoacuity test as a screening tool. The Randot stereotest is a simple and portable tool that can easily be transported to different screening venues and this should motivate clinicians and researchers to perform more comprehensive screenings. However, further studies to support some of the findings of this study are necessary.

\section{Conclusion}

1. Refractive error, accommodative or vergence anomalies were more likely to present with reduced stereoacuity than cases without the respective anomaly.

2. Those with uncorrected refractive errors or accommodative anomalies had more reduced stereoacuity than those with vergence anomalies. (Possibly severity and type of vergence anomaly should be issues to consider in interpretation of this finding.)

The findings of this study suggest that a simple Randot stereotest could be useful to identify cases of uncorrected refractive error, or accommodative or vergence anomalies from those without such anomalies. Thus, the study extends knowledge regarding the possible use of the Randot stereotest as an indirect tool to screen for refractive, accommodative and vergence anomalies in high school children.

\section{Acknowledgements Competing interests}

The authors declare that they have no financial or personal relationships that may have inappropriately influenced them in writing this article.

\section{Authors' contributions}

S.O.W. was responsible for the conceptualisation, investigation, methodology, writing of the original draft and review and editing of the article. R.H. was responsible for the supervision of the study.

\section{References}

1. Saladin JJ. Effects of heterophoria on stereopsis. Optom Vis Sci. 1995;72:487-492. https://doi.org/10.1097/00006324-199507000-00008

2. Saladin JJ. Stereopsis from a performance perspective. Optom Vis Sci. 2005;82:186-205. https://doi.org/10.1097/01.0PX.0000156320.71949.9D

3. Garzia R. The relationship between visual efficiency problems and learning. In: Scheiman M, Rouse M, editors. Optometric management of learning-related vision problems. 1st ed. St. Louis, MO: Mosby, 2006; p. 209-280.

4. Scheiman M, Wick B. Clinical management of binocular vision: Heterophoric, accommodative and eye movement disorders. 3rd ed. Philadelphia, PA: JB Lippincott; 2008 . 
5. Wajuihian SO, Hansraj R. Vergence anomalies in a sample of high school children in South Africa. J Optometry. 2015:12:pii: S1888-4296 (15)00085-0. https://doi. in South Africa. J Optometry. 2015:12010.006
org/10.1016/j.optom.2015.10.006

6. Ponsonby AL, Smith K, Williamson E, Bridge D, Carmichael A. Poor stereoacuity among children with poor literacy: Prevalence and associated factors. Optom Vis Sci. 2013;1:75-83. https://doi.org/10.1097/OPX.0b013e3182780dd0

7. Castren JH. Rutanen AJ. On the significance of heterophoria for stereoscopic vision. Aviat Space Environ Med. 1982;53:393-397.

8. Yang S, Sheedy JE. Effects of vergence and accommodative responses on viewer's comfort in viewing 3D stimuli. Proceedings of the SPIE 7863. Stereoscopic Displays Appl. 2011;XXII:78630Q. https://doi.org/10.1117/12.872546

9. Read JC. What is stereoscopic vision good for? Proceedings of the SPIE 9391. Stereoscopic Displays Appl. 2015;xxvi:93910n.

10. Ju-Wen Y, Tien-Yi H, Ko-Jen Y, Jiahn-Shing L, Wan-Chen K, Ling Y. The effects of hyperopic and astigmatic ametropia on stereoacuity by Titmus stereo test. Taiwan J Ophthalmol. 2012;2:22-24. https://doi.org/10.1016/j.tjo.2011.11.004

11. Castren J, Stjernschantz J, Ario J. Heterophoria- its influence on stereopsis and the importance of cycloplegia in refraction testing of pilot applicants. Aviat Space Environ Med. 1978;49:737-741.

12. Trachimowicz R, Dat BS, Steele G. The effects of uncorrected refractive errors on three tests of stereopsis. Optom Vis Sci. 2000;77:271. https://doi.org/10.1097/ 00006324-200012001-00447

13. Ip JM, Robaei D, Kifley A, Wang JJ, Rose KA, Mitchel P. Prevalence of hyperopia and association with eye findings in 6 and 12 -years olds. Ophthalmology 2008;115:678-685. https://doi.org/10.1016/j.ophtha.2007.04.061

14. Kulp MT, Ying G, Huang J, et al. Associations between hyperopia and other vision and refractive error characteristics. Optom Vis Sci. 2014;91:383-389. https://doi. org/10.1097/OPX.0000000000000223

15. Yang J, Tien-Yi H, Jiahn-Shing L, Yeung L, Yi-Fang L, Chi-Chin S. Correlation between myopic ametropia and stereoacuity in school-aged children in Taiwan. Jpn Ophthalmol. 2013;57:316-319. https://doi.org/10.1007/s10384-013-0231-2

16. Moghadam HM, Ansarei H, Yekta A, Sargolzaei M. The effect of anisometropia on stereopsis. Shahrekord Univ Med Sci. 2008;10:52-58.

17. Mortazavi AA, Ashtari A, Aliyai MN. Comparison of the correlation between refractive errors (myopia \& hyperopia) with stereopsis. J Semnan Univ Med Sci. 2006;7:7-12.

18. Robaei D, Huynh SC, Kifley A, Gole GA, Mitchell P. Stereoacuity and ocular associations at age 12 years: Findings from a population-based study. J AAPOS. 2007;11:356-361. https://doi.org/10.1016/j.jaapos.2006.11.111

19. Gawecki M, Adamski J. Anisometropia and stereopsis. Klin Oczna. 2004;106:561563.

20. Xiaoyi G, Yuan Y, Xianqun F, Xing X. A survey of refractive status and stereopsis in school-aged children in Shanghai. Chinese J Optom Ophthal. 2014;16:132-136.

21. Larson WL, Lachance A. Stereoscopic acuity with induced refractive errors. Am J Optom Physiol Optics. 1983;60:509-551. https://doi.org/10.1097/00006324198306000-00011

22. Wu WW, Peng XJ. Anisometropia and stereopsis. Guoji Yanke Zazhi Int Eye Sci J. 2014;14:74-76.

23. Garzia RP, Nicholson SB. A study of binocular accommodative and vergence facility and predictive analysis of global stereopsis. J Behav Optom. 1991;2:3-6.

24. Shippman S, Kenneth R, Cohen MD. Relationship of heterophoria to stereopsis. Arch Ophthalmol. 1983;101:609-610. https://doi.org/10.1001/archopht.1983. 01040010609017

25. Rosenfield M, Hue JE, Rae R, Yuliya B. The effects of induced oblique astigmatism on symptoms and reading performance while viewing a computer screen. Ophthal PhysiolOpt.2012;32:142-148.https://doi.org/10.1111/j.1475-1313.2011.00887.x

26. Wajuihian SO, Hansraj R. Prevalence of accommodative anomalies in a sample of Black high school children in South Africa. Ophthalmic Epidemiol. 2016;23(5):316323. https://doi.org/10.3109/09286586.2016.1155715
27. Wajuihian SO, Hansraj R. Association of symptoms with refractive, accommodative and vergence anomalies in high school children in South Africa. Optom Vis Perf. 2017;5(1):27-36.

28. Shin HS, Park SC, Park CM. Relationship between accommodative and vergence dysfunctions and academic achievement for primary school children. Ophthal Physiol Opt. 2009;29:615-624. https://doi.org/10.1111/j.1475-1313. 2009.00684.x

29. Bade A, Boas M, Gallaway M, Mitchell L, Scheiman M, Kulp MT. Relationship between clinical signs and symptoms of convergence insufficiency. Optom Vis Sci. 2013;90:988-995. https://doi.org/10.1097/OPX.0000000000000012

30. Ciner EB, Ying GS, Kulp MT, et al. Stereoacuity of preschool children with and without vision disorders. Optometry Vis Sci. 2014;91:351-358. https://doi. org/10.1097/OPX.0000000000000165

31. Hoffman DM, Girshick AR, Akeley K, Banks MS. Vergence-accommodation conflicts hinder visual performance and cause visual fatigue. J Vis. 2014;8:1-30. https://doi.org/10.1167/8.3.33

32. Daum KM. Accommodative dysfunction. Doc Ophthalmol. 1983;55:177-198. https://doi.org/10.1007/BF00140808

33. Goss DA, Zhai H. Clinical and laboratory investigations of the relationship of accommodation and convergence function with refractive error: A literature review. Doc Ophthalmol. 1994;86:349-380. https://doi.org/10.1007/BF01204595

34. Cooper J, Jamal N. Convergence insufficiency-a major review. Optometry 2012;83:137-158.

35. American Optometric Association. Optometric clinical practice guidelines: Care of the patient with accommodative and vergence dysfunctions [homepage on the Internet]. 2013 cited [2017 Jan 15]. Available from: http://www.aoa.org/ documents/CPG-20

36. Von Noorden G, Campos E. Binocular vision and ocular motility: Theory and management of strabismus. 6th ed. St Louis, MO: Mosby; 2002.

37. Lugtigheid AJ, Wilcox LM Allison RS, Howard IP. Vergence eye movements are not essential for stereoscopic depth. Proc Biol Sci. 2014;281:20132118. https://doi. org/10.1098/rspb.2013.2118

38. Ukwade M, Bedell H, Harwerth R. Stereopsis is perturbed by vergence error. Vis Res. 2003;43:181-193. https://doi.org/10.1016/S0042-6989(02)00408-X

39. Ukwade $M$, Bedell $H$, Harwerth R. Stereothresholds with simulated vergence variability and constant error. Vis Res. 2003;43:195-204. https://doi.org/10.1016/ S0042-6989(02)00409-1

40. Jones R. Anomalies of disparity detection in the human visual system. J Physiol. 1977;264:621-640. https://doi.org/10.1113/jphysiol.1977.sp011686

41. Giaschi DE, Narasimhan S, Solski T, Harrison E, Wilcox L. On the typical development of stereopsis: Fine and coarse processing. Vis Res. 2013;89:65-71. https://doi.org/10.1016/j.visres.2013.07.011

42. Laird PW, Hatt SR, Leske DA, Holmes JM. Distance stereoacuity in prism induced convergence stress. J AAPOS. 2008;12:370. https://doi.org/10.1016/j.jaapos. 2008.01.013

43. Dowley D. Heterophoria. Optom Vis Sci. 1990;67:456-460. https://doi. org/10.1097/00006324-199006000-00010

44. Thorn F, Gwiazda J, Cruz AV, Bauer J, Held R. The development of eye alignment, convergence, and sensory binocularity in young infants. Invest Ophthalmol Vis Sci. 1994;35:544-553.

45. Bai-Chuan J. Parameters of accommodative and vergence systems and the development of late-onset myopia. Invest Ophthalmol Vis Sci. 1995;36:17371742.

46. Fielder AR, Moseley MJ. Does stereopsis matter in humans? Eye 1996;10:233238. https://doi.org/10.1038/eye.1996.51

47. Wajuihian SO, Hansraj R. Near vision anomalies in Black high school children in Empangeni, South Africa: A pilot study. Afr Vis Eye Health. 2014;73:21-38. https:// doi.org/10.4102/aveh.v73i1.4 